\title{
A singular perturbation method for saddle connections and subharmonics of certain nonlinear differential equations with fixed saddle points
}

\author{
Peter SMith
}

\begin{abstract}
Saddle connections and subharmonics are investigated for a class of forced second order differential equations which have a fixed sadile point. In these equations, which have linear damping and a nonlinear restoring term, the amplitude of the forcing term depends on displacement in the system. Saddle connections are significant in nonlinear systems since their appearance signals a homoclinic bifurcation. The approach uses a singular perturbation method which has a fairly broad application to saddle connections and also to various subharmonics. The singular perturbation is unusual in that it uses a time-scale which has to be constructed over an infinite interval. The system with a cubic restoring term and a quadratic amplitude is looked at in some detail.
\end{abstract}

\section{INTRODUCTION}

For certain nonlinear differential equations homoclinic bifurcation occurs when the stable and unstable manifolds of a saddle point of the system intersect thus creating what is known as a homoclinic saddle connection. Probably the most widely investigated differential equation which exhibits homoclinic bifurcation, period doubling and strange attractors is Duffing's equation with linear damping, cubic restoring force and harmonic forcing. The qualitative behaviour of solutions of this equation is now well understood and a comprehensive account of the subject can be found in the text by Guckenheimer and Holmes [6]. The review by Greenspan and Holmes [5] and the book by Thompson and Stewart [13] contain further background material relevant to the subject of this paper.

In Duffing's equation the saddle point is associated with the Poincare map of the fixed point of an unstable periodic solution. The location of this fixed point varies in the parameter space of the system. From the point of view of applying perturbation procedures there is considerable insight to be gained by

1980 Mathematics Subject Classification (1985 revision): 34D15, 58F22. Editorial de la Universidad Complutense. Madrid, 1990. 
investigating model systems which have fixed saddle points. An equation which can be made to have this type of fixed point is

$$
\ddot{x}+k \dot{x}+f(x)+\mu g(x) \cos \omega t=0
$$

where $k$ is the damping coefficient, $\omega$ is the forcing frequency and $\mu$ can be thought of as a measure of the forcing amplitude. We assume that

$f, g \in C^{\prime}(-\infty, \infty), f(0)=g(0)=0, f(x)=-x+o(x)$ and $g(x)=-\delta x-x^{2}+o\left(x^{2}\right)$ and that $f(x)$ is an odd function of $x$ such that $f(x) \rightarrow \infty$ as $x \rightarrow \infty$. We also assume that $f(x)$ has just one stationary value, which is a minimum, for $x>0$. In equation (I) the parameters are such that $k=k_{0} \in$ and $\mu=\mu_{0} \epsilon$ where $k_{0}$, $\mu_{0}=\mathrm{O}(1)$ and $\epsilon$ is a small parameter. Essentially we intend to treat the equation as a perturbation of the autonomous system

$$
\ddot{x}+f(x)=0 .
$$

Equation (1) is a version of Duffing's equation if $g(x)=1$, but, in this form, the permanent equilibrium point at the origin is lost. It can also be viewed as an approximation to Duffing's equation in which the unstable periodic solution is removed by a perturbation of its averaged form (see Smith and Davenport [12] for this derivation).

If $g(x)=x$ then the equation becomes a damped Mathieu equation with cubic restoring force. Other versions of equation (1) arise in circuit theory (see, for example, the work of Salam and Sastry [11], Hasler [7] and Endo and Chua [4]), where there is considerable interest in chaotic outputs from systems with particular reference to Josephson junction devices and phase locked loops.

The purpose of the present paper is to investigate the structure of saddle connections and subharmonics of (1) as a contribution to nature of the chaotic regime of the system. It is part of a continuing programme of work initiated by the paper by the author and Davenport [12].

\section{MELNIKOV'S METHOD}

We first need to establish the behaviour of solutions of (1) in the neighbourhood of the origin. If $g(x)=-x^{2}+o\left(x^{2}\right)(\delta=0)$, then sufficiently close to the origin for $\epsilon$ small, equation (i) can be approximated by

$$
\ddot{x}+\epsilon k_{0} \dot{x}-x=0 \text {. }
$$

The equilibrium point at the origin is always a fixed saddle point. With any saddle point we can associate stable and unstable manifolds which are 
sets of points or initial values whose first returns or Poincaré maps, period $2 \pi / \omega$, approach the saddle point for iterations forward and backward in time respectively.

When $\delta=0$ as in (3), the stable and unstable manifolds approach the origin asymptotically along the straight lines $y=\left(-1-\frac{1}{2} \epsilon k_{0}\right) x$ and $y=\left(1-\frac{1}{2} \epsilon k_{0}\right) x$ respectively, for small $\epsilon$. Timewise $x$ must behave as $x \sim e^{\left(t-1-1_{2}+k_{0}\right) t}$ and $x \sim e^{\left(t-\frac{1}{2} \cdot k_{0}\right) r}$ as $t \rightarrow \infty$ and $t \rightarrow-\infty$ respectively.

If $g(x)=-x+o(x)$ (case, $\delta=1$ ), then the linearised equation close to the origin becomes

$$
\ddot{x}+\epsilon k_{0} \dot{x}-\left(1-\epsilon \mu_{0} \cos \omega t\right) x=0,
$$

which is the damped Mathieu equation. Floquet theory (see Jordan and Smith [9]) indicates that the solutions of (3) are of the form

$$
x=c_{1} e^{\left(0-1_{2} \in k_{0}\right) t} p_{1}(t)+c_{2} e^{-\left(0+\frac{1}{2} * k_{0}\right) t} p_{2}(t)
$$

where $\pm \sigma$ are the characteristic exponents, $p_{1}(t)$ and $p_{2}(t)$ are periodic functions with minimal period $2 \pi / \omega$ and $c_{1}$ and $c_{2}$ are constants. It is evident from (4) that the origin is still a saddle point for $\epsilon$ sufficiently small, with the asymptotic behaviour of the stable and unstable manifolds given by putting successively $c_{1}=0$ and $c_{2}=0$ in (5).

The method due to Melnikov [10] (see also Holmes [8] and Guckenheimer and Holmes [6] for particular references to Duffing's equation) is a perturbation procedure which determines the parameter values for which intersections ot the stable and unstable manifolds occur. Any such intersection signals the appearance of a saddle connection for the saddle point.

Let $\dot{x}=y$ and put

$$
\dot{\mathbf{x}}=\left[\begin{array}{l}
\dot{x} \\
\dot{y}
\end{array}\right]=\left[\begin{array}{c}
y \\
-f(x)-\epsilon\left(k_{0} y+\mu_{0} g(x) \cos \omega t\right)
\end{array}\right]=\mathbf{f}(\mathbf{x})+\epsilon \mathbf{g}(\mathbf{x}, t),
$$

say. The Melnikov function associated with (6) is defined by

$$
M\left(\bar{t}_{0}\right)=\int_{-\infty}^{\infty} \mathbf{f}\left(\mathbf{x}_{0}(t)\right) \Lambda \mathbf{g}\left(\mathbf{x}_{0}(t), t+\bar{t}_{0}\right) d t
$$

where $\mathbf{f} \Lambda \mathbf{g}$ means the wedge product $f_{1} g_{2}-f_{2} g_{1}$ and $\mathbf{x}_{0}(t)=\left[x_{0}(t), y_{0}(t)\right]^{t}$ is the homoclinic orbit or separatrix of the unperturbed system (2). The time $\bar{t}_{0} \in[0,2 \pi / \omega)$ arises since the unperturbed solution $x_{0}(t)$ can always contain an arbitrary time translation. The function defined by $(7)$ is a measure of the distance between manifolds. If there exist of parameter values for $(k, \mu, \omega)$ for 
which $M\left(\bar{t}_{0}\right)$ vanishes, then, for $\epsilon$ sufficiently small, the stable and unstable manifolds intersect and each intersection generates a saddle connection. Substitution of (6) into (7) leads to

$$
M\left(\bar{t}_{0}\right)=-\int_{-\infty}^{\infty} y_{0}(t)\left[k_{0} y_{0}(t)+\mu_{0} g\left(x_{0}(t)\right) \cos \omega\left(t+\tilde{t}_{0}\right)\right] d t .
$$

We can illustrate the theory using two examples. In both cases $f(x)=-x+x^{3}$ and $x_{0}(t)=\sqrt{2} \operatorname{sech} t$.

Example 1. $(\delta=1)$. If $g(x)=-x$, then the Melnikov function becomes

$$
M\left(\bar{t}_{0}\right)=-\frac{4}{3} k_{0}+\pi \omega^{2} \mu_{0} \operatorname{cosech}\left(\frac{1}{2} \omega \pi\right) \sin \omega \bar{t}_{0} .
$$

Saddle connections can only occur if

$$
\frac{k}{\mu} \leq \frac{3}{4} \pi \omega^{2} \operatorname{cosech}\left(\frac{1}{2} \omega \pi\right) .
$$

Example 2. $(\delta=0)$, If $g(x)=-x^{2}$, then

$$
M\left(\bar{t}_{0}\right)=-\frac{4}{3} k_{0}+\frac{1}{3} \sqrt{2} \mu_{0} \pi \omega\left(\omega^{2}+1\right) \operatorname{sech}\left(\frac{1}{2} \omega \pi\right) \sin \omega \bar{t}_{0}
$$

in which case saddle connections only exist if

$$
\frac{k}{\mu} \leq \frac{1}{4} \sqrt{2} \pi \omega\left(\omega^{2}+1\right) \operatorname{sech}\left(\frac{1}{2} \omega \pi\right) .
$$

In both cases $k / \mu$ has to be below a critical threshold for homoclinic saddle connections to appear.

\section{SINGULAR PERTURBATION APPROACH}

We propose to construct a solution using the coordinate perturbation procedure developed by Crocco [3] (see also Jordan and Smith [9], Chapter 6)). This will be a singular perturbation of a separatrix solution valid on $t \in(-\infty, \infty)$.

Let the right-hand separatrix of the unperturbed system (2) cut the $x$-axis in the phase plane at $x=\alpha_{0}$, where $\alpha_{0}$ must be the positive solution of

$$
\int_{0}^{\alpha_{0}} f(x) d x=0 .
$$

For the autonomous case the time of intersection of the separatrix with the $x$-axis at $\alpha_{0}$ is immaterial, but it is significant for the nonautonomous system when we need to know which particular separatrix solution is going to be the 
first approximation to a saddle connection. Our approach requires the analysis of those solutions which start close to $\left(\alpha_{0}, 0\right)$ at time of approximately $t_{0}$, which is not known a priori, but will be defined by the required behaviour of the solutions as $t \rightarrow \pm \infty$.

Consider now those solutions of (1) which start or end at $x=\alpha_{0}+\epsilon \alpha_{1}+\ldots$, $\dot{x}=0$ at time $t=t_{0}+\epsilon t_{1}+\ldots$. Both forward and backward in time these initial conditions become

$$
x\left(t_{0}+\epsilon t_{1}+\ldots, \epsilon\right)=\alpha_{0}+\epsilon \alpha_{1}+\ldots, \quad x\left(t_{0}+\epsilon t_{1}+\ldots, \epsilon\right)=0 .
$$

The introduction of the expansion

$$
x(t, \epsilon)=x_{0}(t)+\epsilon x_{1}(t)+\ldots
$$

into (1) leads to a sequence of differential equations of which the first two are

$$
\begin{gathered}
\ddot{x}_{0}+f\left(x_{0}\right)=0, \\
\ddot{x}_{\mathrm{t}}+f\left(x_{0}\right) x_{1}=-k_{0} x_{0}-\mu g\left(x_{0}\right) \cos \omega t,
\end{gathered}
$$

subject to the initial conditions

$$
\begin{gathered}
x_{0}\left(t_{0}\right)=\alpha_{0}, \quad x_{0}\left(t_{0}\right)=0, \\
x_{1}\left(t_{0}\right)=\alpha_{1}, \quad x_{1}\left(t_{0}\right)=-t_{1} \ddot{x}_{0}\left(t_{0}\right)
\end{gathered}
$$

The general solution of (15) can be expressed in terms of the separatrix solution $x_{0}(t)$ of $(14)$ : it is

$$
x_{1}(t)=A u_{1}(t)+B u_{2}(t)+\int_{t_{0}}^{t}\left[u_{1}(s) u_{2}(t)-u_{2}(s) u_{1}(t)\right] q(s) d s
$$

where

$$
u_{1}(t)=x_{0}(t), \quad u_{2}(t)=u_{1}(t) \int \frac{d t}{\left[u_{1}(t)\right]^{2}},
$$

and

$$
q(t)=-k_{0} x_{0}(t)-\mu g\left[x_{0}(t)\right] \cos \omega t .
$$

The constants $A$ and $B$ as determined by the initial conditions are given by

$$
A=-t_{1}, \quad B K=\alpha_{1},
$$


where

$$
K=\lim _{t \rightarrow t_{0}} u_{2}(t)
$$

The asymptotic behaviour of the homogeneous part of equation (15) is covered by a theorem due to Bellman [1] (see also Cesari [2], Section 5.4). This is justified since the conditions

$$
1+f^{\prime}\left(x_{0}\right) \rightarrow 0 \text { as } t \rightarrow \pm \infty \text { and } \int_{0}^{t}\left[1+f^{\prime}\left(x_{0}\right)\right]^{2} d s<\infty
$$

hold. Bellman's theorem then states that the homogeneous part of the solution of (15) behaves as $e^{ \pm(i+0(1))}$ for both $t \rightarrow \pm \infty$. These solutions can be identified with $u_{1}(t)$ and $u_{2}(t)$ in (19).

It is a direct consequence of this asymptotic behaviour that secular terms (that is, those behaving as $t$ or $\cos \omega t$ as $t \rightarrow \pm \infty$ ) will arise from the second integral in (18). They appear specifically from the integration of the product of $u_{2}(s)$ and any linear terms in $x_{0}$ and $\dot{x}_{0}$ in $q(t)$. Such terms can be eliminated by a suitable coordinate perturbation in the form

$$
t=\tau+\epsilon T_{1}(\tau)+\ldots
$$

In terms of the new time scale $\tau$ the perturbation becomes

$$
\begin{aligned}
x=X(\tau, \epsilon) & =x_{0}\left(\tau+\epsilon T_{1}(\tau)+\ldots\right)+\epsilon x_{1}\left(\tau+\epsilon T_{1}(\tau)+\ldots\right)+\ldots, \\
& =x_{0}(\tau)+\left[T_{1}(\tau) x_{0}^{\prime}(\tau)+x_{1}(\tau)\right] \epsilon+\ldots
\end{aligned}
$$

where $x_{0}^{\prime}(\tau)=d x_{0}(\tau) / d \tau$. We now choose $T_{1}(\tau)$ to take out the secular expression in the second term of the expansion: thus we put

$$
T_{1}(\tau)=-\int_{t_{0}}^{\tau} u_{2}(s)\left[k_{0} \dot{x}_{0}(s)-\mu \delta x_{0}(s) \cos \omega s\right] d s .
$$

With this correction the expansion finally becomes

$$
\begin{aligned}
& x=X(\tau, \epsilon)=x_{0}(\tau)+\epsilon\left[-t_{1} u_{1}(\tau)+\left(\frac{\alpha_{1}}{K}\right) u_{2}(\tau)\right. \\
& +u_{2}(\tau) \int_{t_{0}}^{\tau} u_{1}(s) q(s) d s+u_{1}(\tau) \int_{t_{0}}^{\tau} u_{2}(s)\left\{\mu g\left[x_{0}(s)\right]\right. \\
& \left.\left.+\mu \delta x_{0}(s) \cos \omega s\right\} d s\right]+\ldots
\end{aligned}
$$

If we assume that $\tau=\tau_{0}+\epsilon \tau_{1}+\ldots$ then $t=t_{0}+\epsilon t_{1}+\ldots$, then from the coordinate perturbation (22)

$$
t_{0}=\tau_{0}, \quad t_{1}=\tau_{1}+\epsilon T_{1}\left(\tau_{0}\right)+\ldots
$$


In the previous approach to Duffing's equation by Smith and Davenport [12], a version of Lighthill's method [9], Section 6.3 was used to obtain a different coordinate perturbation.

\section{SADDLE CONNECTIONS}

We can treat (17) and (18) as uinitialn conditions both forward and backward in time. The forward solution approaches the saddle if $x(t, \epsilon) \rightarrow 0$ and $x(t, \epsilon) \rightarrow 0$ as $t \rightarrow \infty$, and the backward solution emanates from the saddle if these limits also hold as $t \rightarrow-\infty$. A saddle connection is formed if all four conditions are met. From the remarks on asymptotic behaviour at the end of the previous section, a saddle connection occurs if both

$$
B+\int_{r_{0}}^{\infty} u_{1}(s) q(s) d s=0
$$

and

$$
B-\int_{-\infty}^{r_{0}} u_{1}(s) q(s) d s=0,
$$

Elimination of $B$ between (25) and (26) leads to the condition

or

$$
\int_{-\infty}^{\infty} u_{1}(s) q(s) d s=0
$$

$$
\int_{-\infty}^{\infty} x_{0}^{\prime}(s)\left\{k_{0} x_{0}^{\prime}(s)+\mu_{0} g\left[x_{0}(s)\right] \cos \omega s\right\} d s=0 .
$$

In the particular case in which $f(x)=-x+x^{3}$ and $g(x)=-x^{2}$, the first term in the expansion is given by $x_{0}(\tau)=\sqrt{2}$ sech $\left(\tau-\tau_{0}\right)$. The zeros of the Melnikov function determined by (10) coincide with those given by (27). When $t_{0}$ is known from (27), $B$ can be found from (25) and $x_{1}$ is then given by (24). The time $\bar{t}_{0}$ which arises in Melnikov's method in Section 2 is the same time as $t_{0}$ or $\tau_{0}$, and $t_{0}$ is the time, to lowest order, when the saddle connection cuts the $x$-axis.

\section{SUBHARMONICS}

The singular perturbation method of the previous section can be adapted to find the location of certain subharmonics of equation (1) when homoclinic bifurcation has taken place. Equation (1) has many characteristics which are similar to those of Duffing's equation and the behaviour of the stable and unstable manifolds as $k / \mu$ decreases through its critical value is very similar 
to those depicted by Guckenheimer and Holmes [6], Section 2.2. Equation (1) develops an attracting set of first returns which includes a period doubling sequence. It is unlikely that the perturbation procedure of Section 4 can be adapted to approximate to the period doubling sequence since these subharmonics grow from the centre of the original unperturbed system.

Orbits which are close to the saddle connection of the previous section will cut either the $x$ or $y$-axes near the origin in the phase plane. A direct perturbation analysis on the autonomous system (2) easily reveals that solutions which pass through $x=\alpha_{0}+\epsilon \alpha_{i}, \dot{x}=0$ whichever occurs first in the neighbourhood of the origin in the phase plane both forward and backward in time. Typical solutions are shown in Figure 1. As $\epsilon \rightarrow 0$, the time interval of this approximation approaches $+\infty$ and the corresponding orbit tends to the separatrix of (2). To simplify the analysis we shall now assume that $\delta=0$ in the definition of $g(x)$.

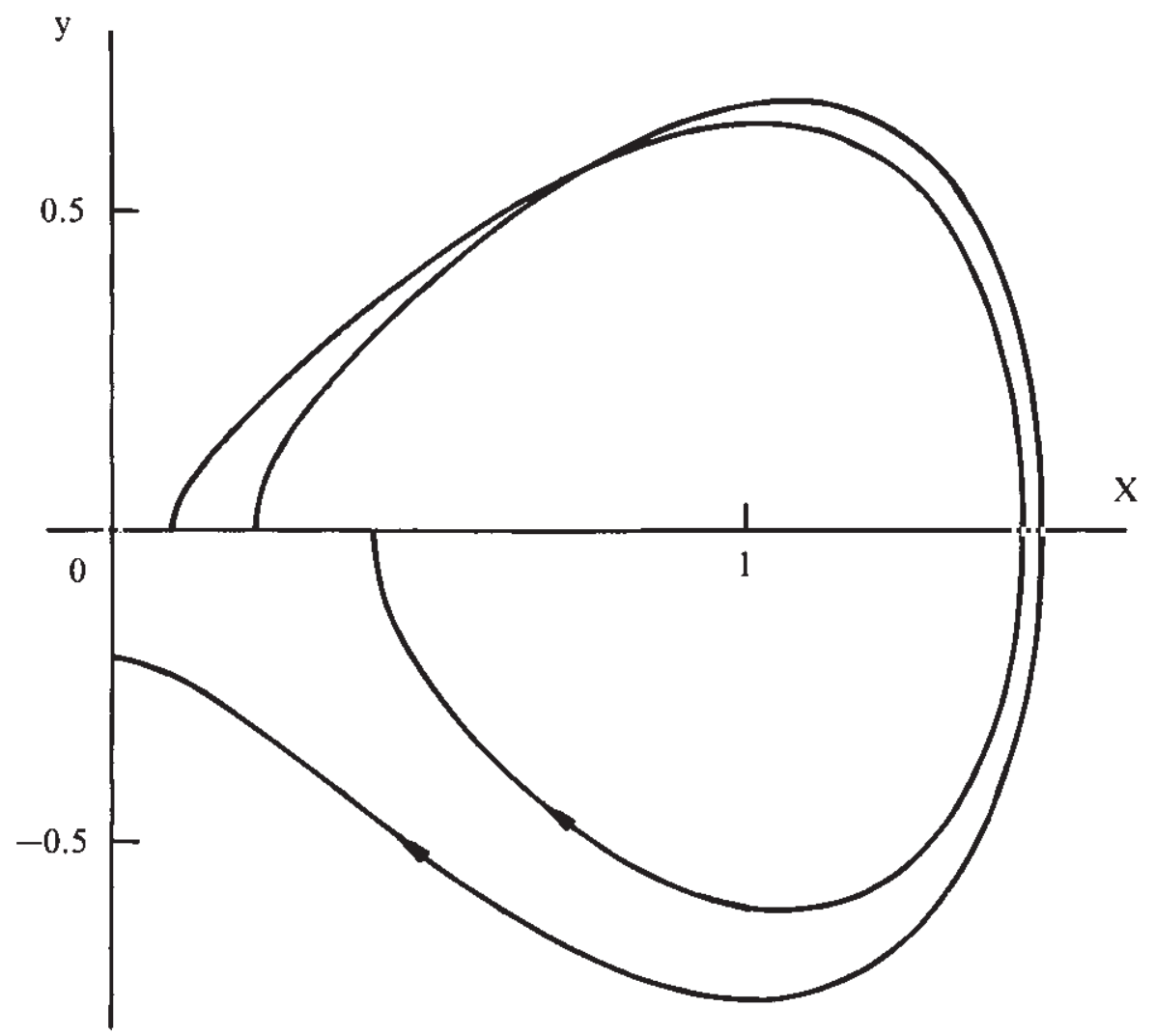

Fig. 1. Orbits which pass close to $x=\sqrt{2}$ for equation (1) with $f(x)=-x+x^{3}$ and $g(x)=-x^{2}$, and parameter values $k=0 \cdot 1, \mu=0 \cdot 2, \omega=1 \cdot 2$. 
Assume also that $x$ is still given by (24), that is, still subject to the initial conditions (16) and (17). The two cases in which the advancing path first meets the $x$-axis and the $y$-axis must be investigated separately. The former case occurs where $t$ satisfies

$$
x(t, \epsilon)=0 .
$$

This time will be a function of $\epsilon$ so that some care is required in assessing the contributions of terms in the expansion of $x(t, \epsilon)$. Equation (28) leads to

$$
x_{0}^{\prime}(\tau)+\epsilon\left[T_{1}(\tau) x_{0}^{\prime \prime}(\tau)+x_{1}^{\prime}(\tau)\right]+\ldots=0 .
$$

Now on the separatrix for $x<0, x_{0}(\tau)$ satisfies

$$
x_{0}^{\prime}(\tau) \approx-x_{0}(\tau)
$$

close to the origin in the phase plane. Thus for $x>0$ and $x<0$ both small in magnitude

$$
u_{1}(\tau) \approx-x_{0}(\tau), \quad u_{2}(\tau) \approx-1 /\left[2 x_{0}(\tau)\right]
$$

Substitution of these approximations and (23) into (29) leads to the result that, for $x_{0}(\tau)$ small,

$$
-x_{0}(\tau)-\frac{1}{2} \epsilon\left\{\left[B+\int_{\tau_{0}}^{\tau} u_{1}(s) q(s) d s\right]\left[x_{0}(\tau)\right]^{-1}+O\left[x_{0}(\tau)\right]\right\}+\ldots=0 .
$$

In the construction of ( 31 ), equation ( 30 ) has been used where necessary. The upper limit of the integral can be extended to infinity since the remainder is $o$ (1) as $\epsilon \rightarrow 0$. If we now put the first two terms of the expansion equal to zero in (3I), then, to lowest order,

$$
x_{0}=\sqrt{ }\left[C_{+}\left(k_{0}, \mu_{0}, \tau_{0}\right) \epsilon\right]+O(1),
$$

where

$$
C_{+}\left(k_{0}, \mu_{0}, t_{0}\right)=-\frac{1}{2}\left\{B+\int_{\tau_{0}}^{\infty} u_{1}(s) q(s) d s\right\}
$$

Such an intersection will only take place if $C_{+}\left(k_{0}, \mu_{0}, \tau_{0}\right)>0$. Furthermore the solution will approach the saddle if $C_{+}\left(k_{0}, \mu_{0}, \tau_{0}\right)=0$ thus agreeing with (25). On the neighbouring stable separatrix

$$
x_{0}(\tau) \sim D_{+} \epsilon^{-\left(\tau-r_{0}\right)},
$$


where $D_{+}$is a known constant. Comparison of (32) with (34) implies that the time lapse between the successive intersections with the $x$-axis is given by

$$
\tau-\tau_{0}=-\frac{1}{2} \ln \epsilon+\ln \left\{D_{+} / \sqrt{ }\left[C_{+}\left(k_{0}, \mu_{0}, \tau_{0}\right)\right]\right\}+o(1) .
$$

By a similar argument the prior intersection with the $x$-axis occurs at

$$
x_{0}=\sqrt{ }\left[C_{-}\left(k_{0}, \mu_{0}, \tau_{0}\right) \epsilon\right]+O(1),
$$

where

$$
C_{-}\left(k_{0}, \mu_{0}, t_{0}\right)=-\frac{1}{2}\left\{B-\int_{-\infty}^{\tau_{0}} u_{1}(s) q(s) d s\right\} .
$$

at time

$$
\tau-\tau_{0}=\frac{1}{2} \ln \epsilon+\ln \left\{D_{-} / \sqrt{ }\left[C_{-}\left(k_{0}, \mu_{0}, \tau_{0}\right) \epsilon\right]\right\}+o(1),
$$

provided, of course, that $C_{-}\left(k_{0}, \mu_{0}, \tau_{0}\right)>0$. In (38) $D_{-}$is given by the asymptotic formula

$$
x_{0}(\tau) \sim D_{-} e^{\tau-\tau_{0}}
$$

on the neighbouring separatix.

In a similar manner we can find the locations of intersections with the $y$ axis in those cases where occur first. At these points in the phase plane

$$
x_{0}^{\prime}(\tau)=\mp \sqrt{ }\left[-C_{ \pm}\left(k_{0}, \mu_{0}, \tau_{0}\right) \epsilon\right]+O(1)
$$

for $y \leqslant 0$.

Subharmonics in $x>0$ can be found by matching (32) and (36) at time differences $2 \pi n / \omega(n=1,2, \ldots)$. This leads to the two conditions

$$
C_{+}\left(k_{0}, \mu_{0}, \tau_{0}\right)=C_{-}\left(k_{0}, \mu_{0}, \tau_{0}\right) \text { or } \int_{-\infty}^{\infty} u_{1}(s) q(s) d s=0 \text {, }
$$

and

$$
2 \pi n=\omega \ln \left[D_{+} D_{-} / \epsilon C_{+}\left(k_{0}, \mu_{0+} \tau_{0}\right)\right] .
$$

Obviously such subharmonics will only be present if $C_{+}, C_{-}>0$. Equation (41) is the same condition as (27) so that these subharmonics will be a consequence of homoclinic bifurcation. 
For the system with $f(x)=-x+x^{3}$ and $g(x)=-x^{2}$ we can find $C_{f}\left(k_{0}, \mu_{0}, \tau_{0}\right)$ explicitly. Since $x_{0}(\tau)=\sqrt{2} \operatorname{sech}\left(\tau-\tau_{0}\right)$, it follows that $D_{+}=D_{-}=2 \sqrt{2}$. Also

$$
u_{1}(\tau)=-\sqrt{2} \sinh \left(\tau-\tau_{0}\right) \operatorname{sech}^{2}\left(\tau-\tau_{0}\right)
$$

and

(44) $\cdot u_{2}(\tau)=\frac{1}{4} \sqrt{2}\left[2-3\left(\tau-\tau_{0}\right) \tanh \left(\tau-\tau_{0}\right)-\sinh ^{2}\left(\tau-\tau_{0}\right)\right] \operatorname{sech}\left(\tau-\tau_{0}\right)$.

The constant $B$ from (21) becomes $B=\alpha_{1} \sqrt{2}$, whilst condition (41) reduces to

$$
\sin \omega \tau_{0}=2 \sqrt{2} k_{0} \cosh \left(\frac{1}{2} \omega \pi\right) /\left[\mu_{0} \omega \pi\left(1-\omega^{2}\right)\right]
$$

as in Section 2. Thus (33) becomes

$$
C_{+}\left(k_{0}, \mu_{0}, \tau_{0}\right)=-\frac{1}{2} \alpha_{1} \sqrt{2}-\frac{1}{2} \int_{0}^{\infty} u_{1}\left(s+\tau_{0}\right) q\left(s+\tau_{0}\right) d s
$$

where

$$
q\left(s+\tau_{0}\right)=\frac{5}{2} \sqrt{2} k_{0} \sinh s \operatorname{sech}^{4} s+2 \mu_{0} \operatorname{sech}^{2} s \cos \omega\left(s+\tau_{0}\right) .
$$

The first term in the integral in (46) is a standard form whilst the second can be transformed by the identity

$$
\begin{gathered}
\sqrt{2} \mu_{0} \int_{0}^{\infty} \sinh s \operatorname{sech}^{4} s \cos \omega\left(s+\tau_{0}\right) d s \\
=\frac{1}{6} \mu_{0} \sqrt{2}\left[\left(2+\omega^{2}\right) \cos \omega \pi_{0}-\omega\left(1+\omega^{2}\right) \int_{0}^{\infty} \operatorname{sech} s \sin \omega\left(s+\tau_{0}\right) d s\right] .
\end{gathered}
$$

In the final integral substitute the series

$$
\operatorname{sech} s=2 \sum_{m=0}^{\infty}(-1)^{m} e^{-(2 m+1) s}, \quad(s>0),
$$

and integrate term-by-term. The result is

(47) $C_{+}\left(k_{0}, \mu_{0}, \tau_{0}\right)=-\frac{1}{2} \alpha_{1} \sqrt{2}+\frac{1}{3} k_{0}+\mu_{0} M(\omega) \cos \tau_{0}+\mu_{0} N(\omega) \sin \omega \tau_{0}$,

where

$$
\begin{gathered}
M(\omega)=\frac{\sqrt{2}}{6}\left[2+\omega^{2}-2 \omega^{2}\left(1+\omega^{2}\right) \sum_{m=0}^{\infty} \frac{(-1)^{m}}{(2 m+1)^{2}+\omega^{2}}\right], \\
N(\omega)=-\frac{\sqrt{2}}{12} \omega \pi\left(1+\omega^{2}\right) \operatorname{sech}\left(\frac{1}{2} \omega \pi\right) .
\end{gathered}
$$


Similarly

$$
C_{-}\left(k_{0}, \mu_{0}, \tau_{0}\right)=-\frac{1}{2} \alpha_{1} \sqrt{2}-\frac{1}{3} k_{0}+\mu_{0} M(\omega) \cos \omega \pi_{0}-\mu_{0} N(\omega) \sin \omega \tau_{0} .
$$

For each positive $n$, equations (41), (42) and (47) now define $\alpha_{1}$, the coordinate of the point where the coresponding subharmonic cuts the $x$-axis. The results are particularly simple for the critical tangency of the manifolds. In this case $\omega \tau_{0}=\frac{1}{2} \pi$ whilst from (42)

$$
\epsilon \alpha_{1}=-8 \sqrt{2} \epsilon^{-2 \pi n / \omega} \text {. }
$$

Note that $\alpha_{1} \rightarrow 0$ as $n \rightarrow \infty$ which implies that the critical saddle connection cuts the axis at $x=\alpha_{0}=\sqrt{2}$ to order $\epsilon$.

These subharmonics have a single cycle in each period unlike the multiple cycling which occurs in the period doubling cascade. Similar subharmonics have been previously discussed in some detail by Greenspan and Holmes [5] who investigated them using a subharmonic Melnikov function. There are differences between the methods and the results which arise because the present approach is based on a perturbation of the separatrix of (1) whilst the Melnikov method used by Greenspan and Holmes uses a periodic orbit of the centre as the starting point.

\section{STABILITY OF SUBHARMONICS}

We are now in a position to test the stability of the subharmonics of the previous section. Suppose that $\alpha_{1}$ and $\tau_{0}$ satisfy

$$
\begin{gathered}
\sin \omega \tau_{0}=2 \sqrt{2} k_{0} \cosh \left(\frac{1}{2} \omega \pi\right) /\left[\mu_{0} \omega \pi\left(1+\omega^{2}\right)\right], \\
-\frac{1}{2} \alpha_{1} \epsilon \sqrt{2}+\epsilon \mu_{0} M(\omega) \cos \omega \tau_{0}=8 e^{-2 \pi n / \omega} .
\end{gathered}
$$

This particular pair $\left(\alpha_{1}, \tau_{0}\right)$ defines the initial state of a subharmonic of order $n$. On $y=0$ perturb the initial state to $\left(\alpha_{1}+p_{0}, \tau_{0}+q_{0}\right)$ at $A$ (see Figure 2), where $\left|p_{0}\right|,\left|q_{0}\right|$ are assumed to be sufficiently small. Suppose that the orbit next cuts the $x$-axis in the neighbourhood of $A$ at $C$ where the displacementtime pair is given by $\left(\alpha_{1}+p_{1}, \tau_{0}+q_{0}\right)$. We now find the linearised relations between the perturbations at $A$ and $C$. From (32) and (47) it is possible to find $x$ at $B$, say $x_{B}$, for $\in$ fixed (but small) and to orders $o\left(p_{0}\right)$ and $o\left(q_{0}\right)$. The result is

$$
x_{B}=A_{n}+\epsilon\left[-\sqrt{2} p_{0}+2\left(\gamma_{1}+\gamma_{2}\right) q\right] /\left(4 A_{n}\right),
$$




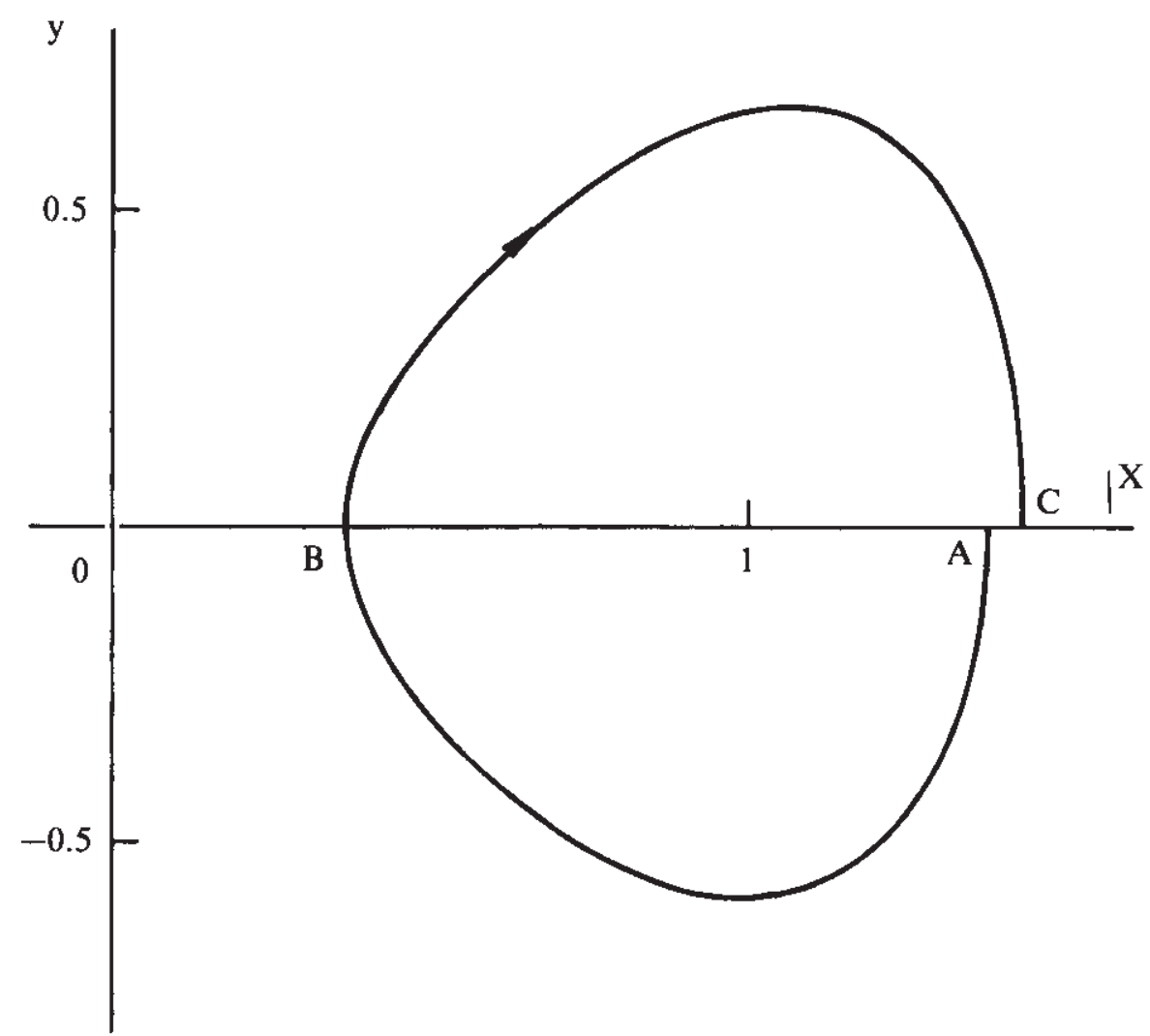

Fig. 2. An orbit close to the theoretical position of the subharmonics for parameter values $k=0 \cdot 2, \mu=0 \cdot 2, \omega=1 \cdot 2$.

where

$$
A_{n}=\sqrt{ }\left[\frac{1}{3} \epsilon k_{0}+8 e^{-2 \pi n: \omega}\right],
$$

$$
\gamma_{1}=-\omega \mu_{0} M(\omega) \sin \omega \tau_{0}, \quad \gamma_{2}=N(\omega) \cos \omega \tau_{0}
$$
that

The prior intersection of the path through $C$ must also pass through $B$ so

$$
x_{B}=A_{n}+\epsilon\left[-\sqrt{2} p_{1}+2\left(\gamma_{1}-\gamma_{2}\right) q_{1}\right] /\left(4 A_{n}\right) .
$$

From (52) and (54) it can be inferred that

$$
-\frac{1}{2} \sqrt{2} p_{1}+\left(\gamma_{1}-\gamma_{2}\right) q_{1}=-\frac{1}{2} \sqrt{2} p_{0}+\left(\gamma_{1}+\gamma_{2}\right) q_{0}
$$


If the path from $A$ arrives at $B$ at a time corresponding to $\tau_{A}$ then, from (35), it follows that

$$
\begin{aligned}
& \tau_{A} \approx \tau_{1}+q_{0}+\ln \left(2 \sqrt{2} / x_{B}\right) \\
& \approx \tau_{0}+\ln \left(2 \sqrt{2} / A_{n}\right)+\left[\frac{1}{2} \sqrt{2} \epsilon p_{0}+\left(2 A_{n}^{2}-\epsilon \gamma_{1}-\epsilon \gamma_{2}\right) q_{0}\right] /\left(2 A_{n}^{2}\right) .
\end{aligned}
$$

Similarly the scaled time $\tau_{A}$ prior to the time at $C$ is given by

$$
\tau_{A} \approx \tau_{0}+(2 \pi n / \omega)-\ln \left(2 \sqrt{2} / A_{n}\right)+\left[-\frac{1}{2} \sqrt{2} \epsilon p_{1}+\left(2 A_{n}^{2}+\epsilon \gamma_{1}-\epsilon \gamma_{2}\right) q_{1}\right] /\left(2 A_{n}^{2}\right) .
$$

Thus, from (56) and (57) with (42),

$$
-\frac{1}{2} \sqrt{2} \epsilon p_{1}+\left(2 A_{n}^{2}+\epsilon \gamma_{1}-\epsilon \gamma_{2}\right) q_{1}=\frac{1}{2} \sqrt{2} \epsilon p_{0}+\left(2 A_{n}^{2}+\epsilon \gamma_{1}-\epsilon \gamma_{2}\right) q_{0} .
$$

Finally, from (55) and (58), the first returns can be expressed by

$$
\mathbf{E}_{1} \mathbf{p}_{1}=\mathbf{E}_{0} \mathbf{p}_{0}
$$

where

$$
\begin{gathered}
\mathbf{p}_{i}=\left[\begin{array}{l}
p_{i} \\
q_{1}
\end{array}\right](i=0,1), \quad \mathbf{E}_{0}=\left[\begin{array}{cc}
-\frac{1}{2} \sqrt{2} \epsilon & \left(\gamma_{1}+\gamma_{2}\right) \epsilon \\
\frac{1}{2} \sqrt{2} \epsilon & 2 A_{n}^{2}-\epsilon \gamma_{1}-\epsilon \gamma_{2}
\end{array}\right] \\
\mathbf{E}_{1}=\left[\begin{array}{cc}
-\frac{1}{2} \sqrt{2} \epsilon & \left(\gamma_{1}-\gamma_{2}\right) \epsilon \\
-\frac{1}{2} \sqrt{2} \epsilon & 2 A_{n}^{2}+\epsilon \gamma_{1}-\epsilon \gamma_{2}
\end{array}\right]
\end{gathered}
$$

The stability of the periodic solutions will depend on the magnitudes of the eigenvalues of the matrix $\mathbf{E}_{1}^{-1} \mathbf{E}_{0}$ in transformation (59). It follows that its eigenvalues $\lambda_{1}, \lambda_{2}$ are the roots of the equation

$$
(\lambda-1)^{2}+2 \lambda \epsilon \gamma_{2} / A_{n}^{2}=0 .
$$

Since $\lambda_{1} \lambda_{2}=1$ and $N(\omega) \neq 0$, one eigenvalue must exceed 1 . Thus $\left(\mathbf{E}_{1}^{-1} \mathbf{E}_{0}\right)^{n}$ will be unbounded for large $n$, and all these subharmonics must be unstable.

\section{LARGE AMPLITUDE SUBHARMONICS}

We can also use the singular perturbation method to search for largeamplitude subharmonics of the same equation. In certain parameter domains 
these solutions appear to be numerically stable over long term runs. Figure 3 shows a period 5 harmonic computed numerically for equation (1). In order to find an approximation to this trajectory we must match solutions which cross the $y$-axis at $B$ and $D$ in Figure 3 at such times as to make the period a multiple of the forcing period. Suppose that the required approximation passes through $A$ at $\tau=\tau_{0}$ and through $C$ at $\tau=\tau_{2}$. From (40) we can find $x_{0}^{\prime}(\tau)$ corresponding to points $B$ and $D$ as they arise from $A$ : they are, say, $y_{B}$ and $y_{D}$, where

$$
\begin{aligned}
& u_{B}=-\sqrt{ }\left[C_{+}\left(k_{0}, \mu_{0}, \tau_{0}\right) \epsilon\right], \\
& y_{D}=\sqrt{ }\left[-C_{-}\left(k_{0}, \mu_{0}, \tau_{0}\right) \epsilon\right]
\end{aligned}
$$

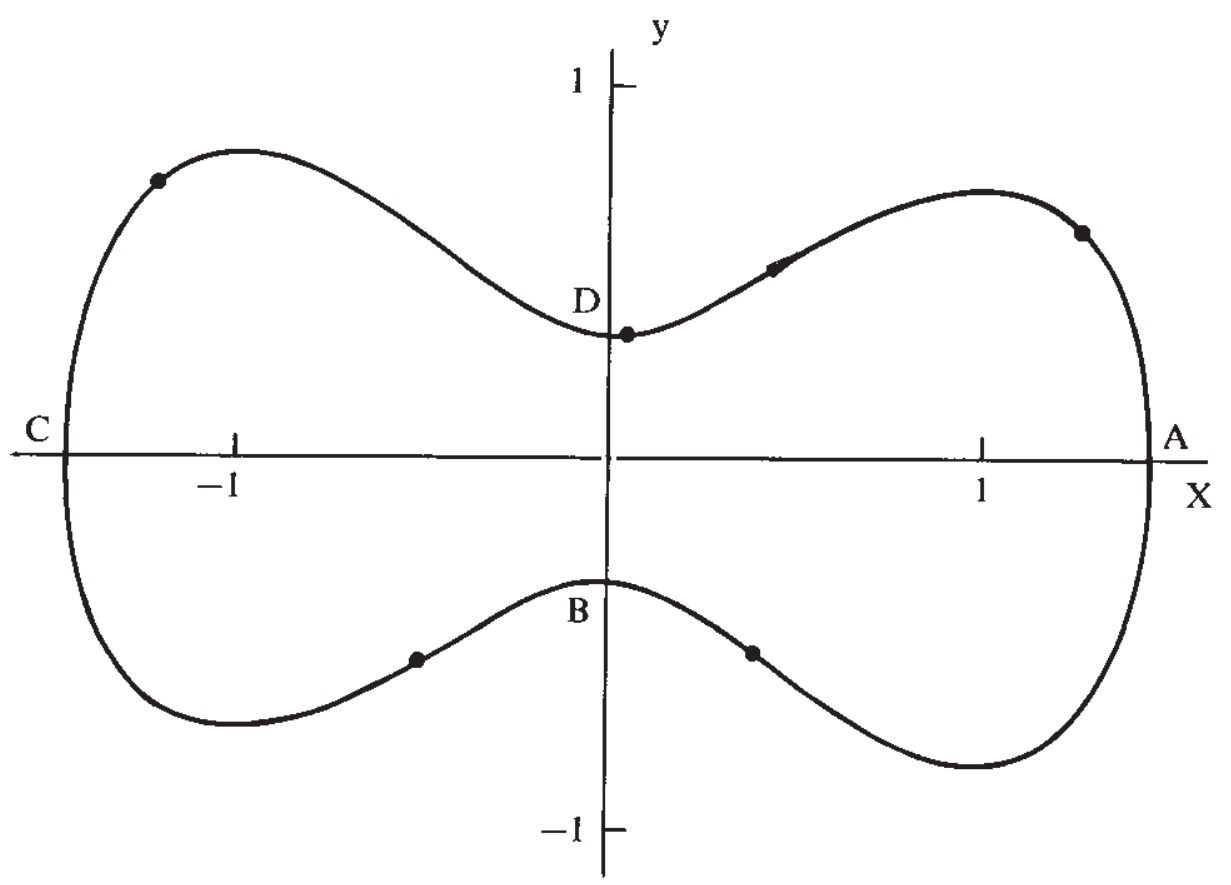

Fig. 3. Period 5 orbit for parameter values $k=0 \cdot 06, \mu=0 \cdot 1, \omega=2 \cdot 8$.

We can construct perturbations for $x<0$ by observing that the original differential equation is unchanged by the mapping $x \rightarrow-x, \mu_{0} \rightarrow-\mu_{0}$. Hence, it follows that $y_{B}$ and $y_{D}$ as generated by initial conditions at $C$ are

$$
\begin{gathered}
y_{B}=-\sqrt{ }\left[-C_{-}\left(k_{0},-\mu_{0}, \tau_{2}\right) \epsilon\right], \\
y_{D}=\sqrt{ }\left[-C_{+}\left(k_{0},-\mu_{0}, \tau_{2}\right) \epsilon\right] .
\end{gathered}
$$


From (60)-(63) it follows that

$$
\begin{aligned}
& C_{+}\left(k_{0}, \mu_{0}, \tau_{0}\right)=C_{-}\left(k_{0},-\mu_{0}, \tau_{2}\right), \\
& C_{-}\left(k_{0}, \mu_{0}, \tau_{0}\right)=C_{+}\left(k_{0},-\mu_{0}, \tau_{2}\right) .
\end{aligned}
$$

We now have to ensure that the closed orbit has a subharmonic period. Let the path start at $D$ and pass through $A, B$ and $C$ at times corresponding to $\tau_{0}, \tau_{1}$ and $\tau_{2}$, and reach $D$ again at time $\tau_{3}$. For $B$ and $D, \tau_{1}$ and $\tau_{3}$ are the actual times whilst $\tau_{0}$ and $\tau_{2}$ correspond to the real times $\tau_{0}-\epsilon T_{1}\left(\tau_{0}\right)$ and $\tau_{2}-\epsilon T_{1}\left(\tau_{2}\right)$. However, to the lowest order we need only consider scaled times.

From (35) and (38), it follows that, approximately,

$$
\tau_{1} \approx \tau_{0}-\frac{1}{2} \ln \epsilon+\ln \left\{D_{+} / \sqrt{ }\left[-C_{+}\left(k_{0}, \mu_{0}, \tau_{0}\right)\right]\right\}
$$

while the initial time at $D$ must be, for periodicity $m, \tau_{3}-2 m \pi / \omega$ where

$$
\tau_{3}=2 m \pi \omega+\tau_{0}+\frac{1}{2} \ln \epsilon-\ln \left\{D_{-} / \sqrt{ }\left[-C_{-}\left(k_{0}, \mu_{0}, \tau_{0}\right)\right]\right\} .
$$

In a similar manner, for $x<0$,

$$
\begin{aligned}
& \tau_{1} \approx \tau_{2}+\frac{1}{2} \ln \left\{D_{+} / \sqrt{ }\left[-C_{-}\left(k_{0},-\mu_{0}, \tau_{2}\right)\right]\right\}, \\
& \tau_{3} \approx \tau_{2}-\frac{1}{2} \ln \left\{D_{-} / \sqrt{ }\left[-C_{+}\left(k_{0},-\mu_{0}, \tau_{2}\right)\right]\right\} .
\end{aligned}
$$

Various relationships between the scaled times can now be found. However, it can be verified that there do exist solutions of (64)-(69) given by

$$
\begin{gathered}
\sin \omega \tau_{0}=-\sin \omega \tau_{2}=k_{0} /\left[3 \mu_{0} N(\omega)\right], \\
\tau_{1}=\frac{1}{2}\left(\tau_{0}+\tau_{2}\right)
\end{gathered}
$$

and

$$
\tau_{3}=\frac{1}{2}\left(\tau_{1}+\tau_{2}\right)+m \pi \omega .
$$

It follows from (70) that we must choose $\omega \tau_{1}$ and $\omega \tau_{2}$ to differ by an odd multiple of $\pi$ for periodicity reasons.

Some numerical computations of these subharmonics can be found in the paper by Greenspan and Holmes [5]. The location of the period three harmonic would indicate that the perturbation procedure is likely to be valid for $m \geq 5$, since the amplitudes of the subharmonics approach $\sqrt{2}$ as $m \rightarrow \infty$. 
There is a consequent narrowing of the attracting set for the higher order subharmonics which makes them increasingly difficult to locate.

The theoretical amplitudes of these subharmonics can be deduced from (66), (70) and (71), from which we infer that

$$
\epsilon \alpha_{1}=\sqrt{2}\left[\mu M(\omega) \cos \omega \tau_{0}+8 e^{-m \pi i \omega}\right], \quad m=3,5,7, \ldots,
$$

where $\omega \tau_{0}$ is given by $(70)$. Numerical computations indicate that only the principal values of $\tau_{0}$ given by $0<\omega \tau_{0}<\frac{1}{2} \pi$ generate stable subharmonics although from the earlier comments on the size of attracting sets this is not conclusive. The theoretical amplitude perturbation versus the forcing amplitude is shown in Figures 4 and 5 for the case $m=5$. The dots are some spot checks on the amplitudes from corresponding numerical computations. The differences between the theoretical and the computed amplitudes is exaggerated by the shortened vertical scales. For smaller values of $\omega$ and larger values $\mu$ either the subharmonics are unstable or their domains of stability are extremely small.

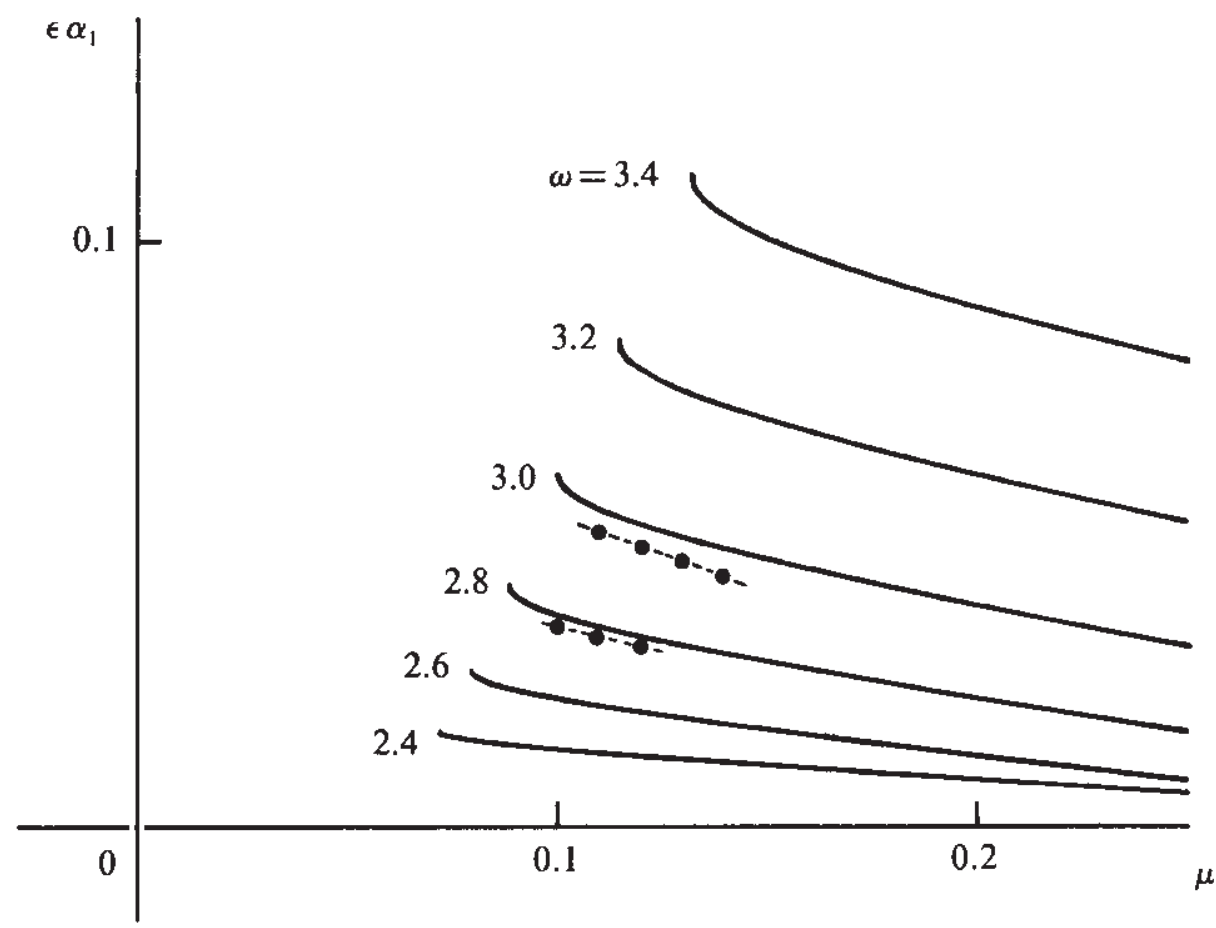

Fig. 4. Theoretical amplitude perturbation $\epsilon \alpha_{1}$ versus forcing amplitude $\mu$ for period five oscillations with $k=0.06$ and $\omega$ in the domain $(2.4,3.4)$. The dots have been obtained from numerically computed amplitudes. 


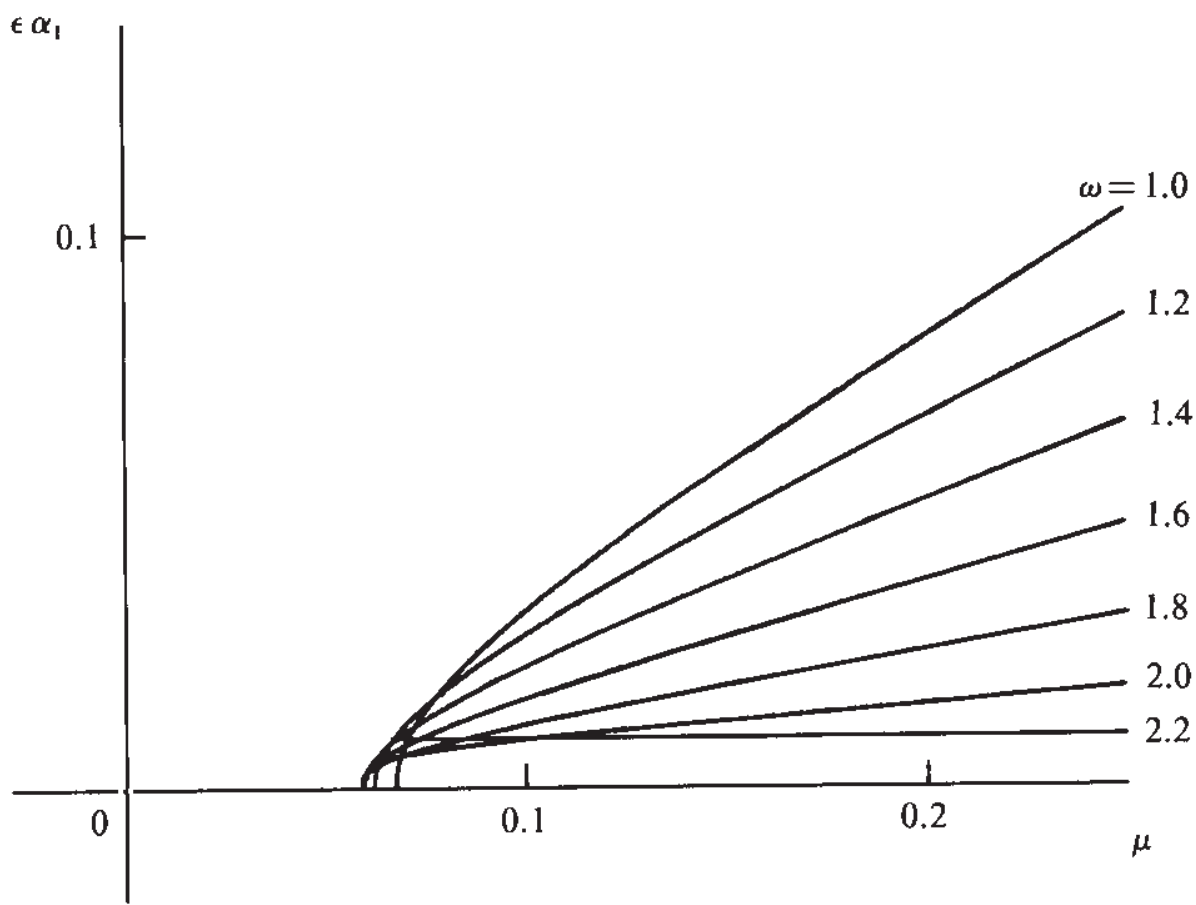

Fig. 5. Theoretical amplitude perturbation $\epsilon \alpha_{1}$ versus forcing amplitude $\mu$ for period five oscillations with $k=0.06$ and $\omega$ in the domain $(1 \cdot 0,2 \cdot 2)$.

\section{CONCLUDING REMARKS}

The singular perturbation method outlined in this paper provides a possible approach to a wide range of initial value problems controlled by equation (1). A particular feature of the approach is its generality since it is possible to obtain an integral solution which does not depend on the precise details of the restoring and amplitude terms. Using this technique a large range of saddle connections and subharmonics can be theoretically determined.

Further developments should include investigations of the bifurcation values of multiple loop and transverse saddle connections of the type obtained for the Duffing oscillator by Smith and Davenport [12], and particularly the stability of large amplitude subharmonics. 


\section{References}

[1] Bellman, R.: On the asymptotic behavior of solutions of $u^{\prime \prime}+(1+f(t)) u=0$. Annali Mat. pura appl. 31 (1950) 83-91.

[2] Cesari, L.: Asymptotic Behavior and Stability Problems in Ordinary Differential Equations. Third edition. Berlin: Springer 1971.

[3] CROCCO, L.: Coordinate perturbations and multiple scales in gas dynamics. Philos. Trans. Roy. Soc. London Ser. A 272 (1972) 275-301

[4] ENDO, T.; ChuA, L. O.: Chaos from phase-locked loops. IEEE Trans. Circuits and Systems. 35 (1988) 987-1003.

[5] GREensPan, B. D.; Holmes, P. J.: Homoclinic orbits, subharmonics and global bifurcations in forced oscillations. Nonlinear Dynamics and Turbulence (edited by G. Barenblatt, G. Ioos and D. D. Joseph), pp. 172-214. Boston: Pitman 1983.

[6] GuCKenheimer, J.; Holmes, P.: Nonlinear Oscillations, Dynamical Systems and Bifurcations of Vector Fields. New York: Springer 1986.

[7] HAsler, J, H.: Electrical circuits with chaotic behaviour. Proc. IEEE. 75 (1987) 1009-1021.

[8] Holmes, P. J.: A nonlinear oscillator with a strange attractor. Philos. Trans. Roy. Soc. London Ser. A 292 (1979) 419-448.

[9] JoRdAN, D. W.; SmITh, P.: Nonlinear Ordinary Differential Equations. Second edition. Oxford: Clarendon Press 1987.

[10] MELNIKOV, V. K.: On the stability of the center for time periodic perturbations. Trans. Moscow Math. Soc. 12 (1963) 1-57

[11] SAl.AM, F. M.; SASTRY, S. S.: Dynamics of the forced Josephson junction circuit: the region of chaos. IEEE Trans. Circuits and Systems. CAS-32 (1985) 784-796.

[12] SMith, P.; DAVENPORT, N. M.: A perturbation method for saddle connections and homoclinic bifurcation in Duffing's equation. Dynamics Stability Systems. 2 (1988) 167-182.

[13] Thompson, J. M. T.; STEWART, H. B.: Nonlinear Dynamics and Chaos. Chichester: Wiley 1986. 